\title{
Confined optical modes in small photonic molecules with semiconductor nanocrystals
}

\author{
Yu. P. Rakovich, ${ }^{\text {a) }}$ M. Gerlach, A. L. Bradley, and J. F. Donegan \\ Semiconductor Photonics Group, Department of Physics, Trinity College, Dublin 2, Ireland
}

T. M. Connolly and J. J. Boland

Department of Chemistry, Trinity College, Dublin 2, Ireland

M. A. Przyjalgowski and A. Ryder

National Centre for Biomedical Engineering Science, National University of Ireland, Galway, Ireland

N. Gaponik

Institute of Physical Chemistry, University of Hamburg, 20146 Hamburg, Germany

\section{A. L. Rogach}

Photonics and Optoelectronics Group, Physics Department and Center for Nanoscience, LudwigMaximilians Universität München, 80799 Munich, Germany

(Received 21 June 2004; accepted 8 September 2004)

\begin{abstract}
We report on the coherent coupling of whispering gallery modes (WGMs) in a photonic molecule formed from two melamine-formaldehyde spherical microcavities coated with a thin shell of light-emitting CdTe nanocrystals (NCs). Utilizing different excitation conditions, the splitting of the WGM resonances originating from bonding and antibonding branches of the photonic states is observed, and fine structure consisting of very sharp peaks resulting from lifting of the WGM degeneracy has been detected. Time-resolved measurements showed a slight increase in the spontaneous emission rate of NCs in a photonic molecule when compared to the spontaneous emission rate for NCs coating a single microsphere. (C) 2004 American Institute of Physics.
\end{abstract}

[DOI: $10.1063 / 1.1812355]$

\section{INTRODUCTION}

In recent years the modification of electromagnetic modes in solid spherical microcavities has been of great interest both for studies of fundamental optical properties and for the potential applications. ${ }^{1,2}$ Extending the ideas of the linear combination of atomic orbitals approach to the classical wave case, it was shown that Mie resonances of single microspheres play the same role as the atomic orbitals in the electronic case. ${ }^{3}$ Following the analogy with quantum mechanics, three integers, $n, l$, and $m$, describing whispering gallery modes (WGMs), correspond to the angular, the radial, and azimuthal quantum numbers, respectively. The spatial distributions of WGM can be described using the eigenfunctions of the vector wave equation for an electromagnetic field in the sphere ${ }^{2}$ by direct analogy with the orbitals of the electron bound in the hydrogen atom as deduced from the Schrödinger equation. This approach has enabled small dielectric spheres to be considered as "photonic atoms." ${ }^{3,4}$ It is well known that the resonant internal field of a spherical cavity is not completely confined to the interior of the microparticle. Depending on the size of the microsphere, the evanescent field can extend into the surroundings up to a couple of micrometers. It was recently recognized that the partial delocalization of Mie resonance states is of great importance because it suggests a possibility for coherent coupling between WGMs of two adjacent spherical particles with closely matched sizes. Such a system of coherently

${ }^{a)}$ Electronic mail: yury.rakovich@tcd.ie coupled photonic atoms may be called a "photonic molecule" (PM) (Ref. 5) and can be employed in order to manipulate photons in the micrometer length scale. In analogy to the formation of molecular electronic orbits, the tight binding approximation provides two combinations for the electromagnetic field in a system of interacting microspheres: bonding (BN) and antibonding (ABN) states. ${ }^{5-8}$ Experimentally, the coupling of the photon modes of individual microspheres in the PM can cause a narrow resonance of a photonic atom to split into two modes of lower quality factor $(Q){ }^{6}$ This phenomenon has been clearly demonstrated in a system of two square, photonic dots coupled by a narrow channel $^{5}$ in a dye-stained bisphere system ${ }^{7,9,10}$ and in chains of polymer-blend microparticles. ${ }^{11}$

Detailed consideration of coherent mode coupling also reveals the possibility of lifting the degeneracy in the PM with respect to $m$ indices. ${ }^{8}$ For the case of off-axis incidence, the modes originating from coupling of WGMs with different $m$ numbers contribute differently to the measured signal, resulting in a number of small peaks ( $m$ resonances) between the upper and lower peaks of $m= \pm 1$. Although theoretically predicted, this phenomenon has not been experimentally observed thus far.

In this paper we have studied the WGM structure in the photoluminescence (PL) spectra of interacting spherical microcavities formed from melamine-formaldehyde (MF) latex microspheres coated with a thin shell of CdTe nanocrystals (NCs) under different excitation conditions. We report on the observation of two major features unique to strong coupling 
between the photonic states of the two spherical microcavities forming the PM. First, we have observed splitting of the WGM resonances originating from $\mathrm{BN}$ and $\mathrm{ABN}$ branches of the photonic states. Secondly, fine structure of very sharp peaks (full width at half maximum $=0.3 \rightarrow 0.5 \mathrm{~nm}$ ) was detected in the PL spectra of the PM, which can be interpreted as a result of the removal of the WGM degeneracy with respect to $m$ due to the difference in symmetry of the PM and the single microsphere.

\section{EXPERIMENT}

Aqueous dispersions of MF microspheres, $2.00 \mu \mathrm{m}$ in diameter with a size deviation of $0.10 \mu \mathrm{m}$ (Microparticles $\mathrm{GmbH}$, Berlin), were combined with luminescent CdTe NCs (Ref. 12) using a layer-by-layer deposition technique, as described elsewhere. ${ }^{13,14}$ The relatively high refractive index $\left(n_{r}=1.68\right)$, high optical transparency, and thermal and mechanical stability of MF make it ideal as a candidate for optical applications, while $\mathrm{CdTe} \mathrm{NCs}$ produce sizedependent emission which can be tuned over a wide spectral region. In this work the colloidal solution of CdTe NCs, with a PL maximum at $620 \mathrm{~nm}$ (2.4-nm radius) and a PL quantum efficiency of $\sim 25 \%$ at room temperature, was used for coating the MF microspheres. The small size of the MF spheres was dictated by specific requirements for the optimal excitation conditions such as matching the laser wavelength to one of the WGM frequencies and achieving good correlation between the WGMs and the laser linewidths. ${ }^{2,13}$ Wide separation of WGM in spheres of this size ${ }^{13}$ avoids intricate band mixing in a PM. Moreover, the moderate quality factor of small spherical particles forming the PM $\left(Q \sim 10^{2}\right.$ for $2-\mu \mathrm{m}$ diameter spheres $\left.{ }^{13}\right)$ provides better mode coupling than would be the case for larger microspheres $\left(Q \sim 10^{3}\right.$ for a 5 - $\mu$-diameter ${ }^{7,15}$ ). To perform an experimental study of the fine structure of coupled WGM, we proceed from the assumption that a stronger coupling parameter can be of crucial importance governing the interaction between electromagnetic fields of two adjacent spheres.

The micro-PL spectra from microspheres were recorded in a backscattering geometry using a RENISHAW microRaman system $\left(1800-\mathrm{mm}^{-1}\right.$ grating, $>1-\mathrm{cm}^{-1}$ spectral resolution) equipped with a notch and plasma filters and a charge-coupled device camera. The spatial resolution of less than $1 \mu \mathrm{m}$ was provided using a microscope with a $\times 100$

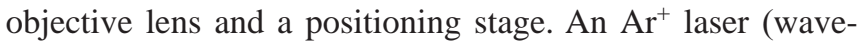
length $\lambda=488 \mathrm{~nm}$ and $1.0 \mathrm{~mW}$ power) was used to provide excitation. For all measurements, a polystyrene/glass substrate was used and two spheres were in contact in order to provide the maximum possible coupling.

Fluorescence lifetimes were measured using a diode laser fluorescence lifetime microscope, which has been previously described. ${ }^{16}$ Briefly, the system comprised of a pulsed diode laser (PicoQuant, LDH-400 laser head and PDL-800B driver), emitting at $405 \mathrm{~nm}$, which has been coupled to an Olympus BX-60 microscope. The scattered excitation light was filtered using a long-pass filter (GG 435LP, AHF) before the fluorescence was focused onto the entrance slit of a $100-\mathrm{mm}$ focal length motorized monochromator (model

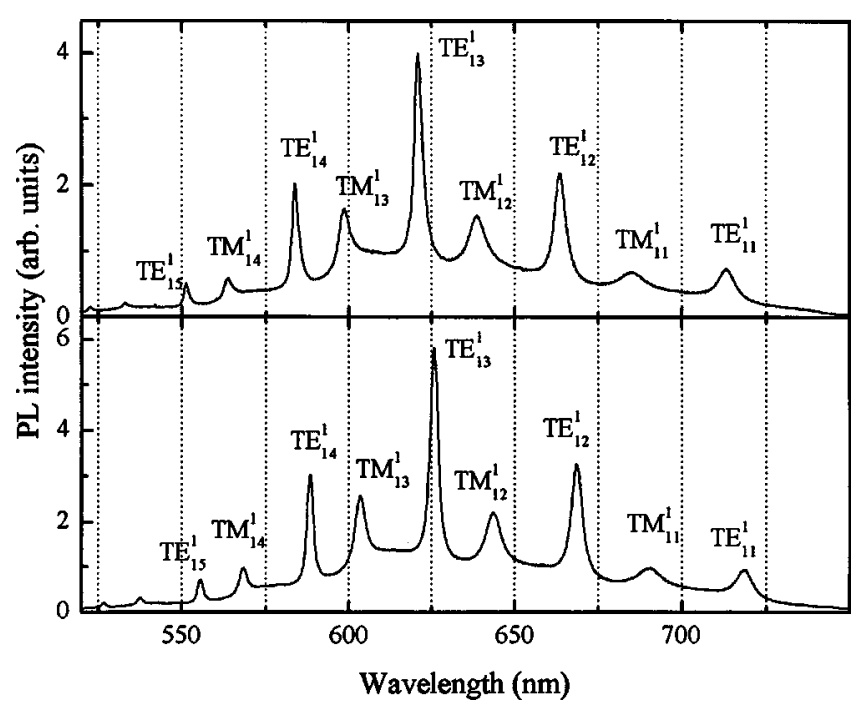

FIG. 1. Emission spectra of two single microspheres with diameters of 1.98 $\mu \mathrm{m}$ (top) and $1.99 \mu \mathrm{m}$ (bottom).

9030, Sciencetech Inc., Canada) fitted with a 1200/mm line grating. The detector was an uncooled metal package photomultiplier tube (model 7353, Hamamatsu) the output of which is fed to the time-correlated single-photon counting electronics (SPC-730 Becker \& Hickl). The instrument response function was measured using a 10-5 M DASPI methanol solution (in a sealed $10-\mathrm{mm}$ path-length quartz cuvette) at the same wavelengths as the microsphere decay curves to discriminate against any monochromator timing effects.

Decays were measured to 10000 counts in the peak and reconvoluted using nonlinear least-squares analysis (FluoFit, PicoQuant), using an equation of the form $I(t) \propto \Sigma_{i} \alpha_{i} \exp$ $\times\left(-t / \tau_{i}\right)$, where $\tau_{i}$ are the PL decay times. The preexponential factors $\alpha_{i}$, where taken into account, were normalized to unity. The quality of the fit was judged in terms of a $\chi^{2}$ value (with a criteria of less than 1.3 for an acceptable fit) and weighted residuals.

\section{RESULTS}

In order to analyze the spectral features of a PM, it is first necessary to examine the PL spectra of single microspheres. Two PL spectra of single microspheres, with slightly different sizes, are shown in Fig. 1. The spectra are essentially identical except for the slight overall shift of the resonances. The observed spectral structure originates from coupling of electronic transitions in NCs to the photon modes of the microsphere, with PL peaks corresponding to the resonant frequencies of WGMs with transverse electric (TE) and transverse magnetic (TM) polarizations. Due to the high PL quantum efficiency of NCs, the peaks in the PL spectra are superimposed on a broad background signal arising from part of the NCs emission, which does not match any WGM of the microsphere.

In the absence of gain, the WGM resonances can be characterized by a mode number $n$ (angular quantum number), mode order $l$ (radial quantum number), and azimuthal mode number $m$ (azimuthal quantum number). The value of 
$n$ is proportional to the circumference divided by the wavelength of the light propagating within the microsphere, the mode order $l$ indicates the number of maxima in the radial distribution of the internal electric field, and the azimuthal mode number $m$ gives the orientation of the WGMs orbital plane. WGMs are degenerate with respect to $m$ because of the spherical symmetry of the sphere.

According to the Lorenz-Mie theory, mathematical conditions for WGM resonances can be given as ${ }^{17}$

$$
n_{r} \psi_{n}(x) \psi_{n}^{\prime}\left(n_{r} x\right)-\psi_{n}\left(n_{r} x\right) \psi_{n}^{\prime}(x)=0 \quad \text { (TM modes), }
$$

and

$$
n_{r} \chi_{n}(x) \psi_{n}^{\prime}\left(n_{r} x\right)-\psi_{n}\left(n_{r} x\right) \chi_{n}^{\prime}(x)=0 \quad \text { (TE modes), }
$$

where $x=\left(2 \pi n_{r} R\right) / \lambda$ is the size parameter, $R$ is the radius of the microsphere, and $\psi_{n}(x)$ and $\chi_{n}(x)$ are the spherical Ricatti-Bessel functions of the first and second kind, respectively.

Solving Eqs. (1) and (2) and comparing the results with the spectral positions of the WGMs in the experimental PL spectra, we can identify the indexes $n$ and $l$ (Fig. 1) for each mode and estimate the relative size of the sphere. Note that a shift in positions of WGMs can be clearly seen between the spectra in Fig. 1. From our calculations, we have determined that this shift is a result of a difference in size of only $14 \mathrm{~nm}$ between the two microspheres.

To investigate the propagation modes in interacting spherical microcavities, we have measured PL spectra by scanning a sample along the longitudinal axis of the PM using excitation/detection geometry as presented in Scheme 1.

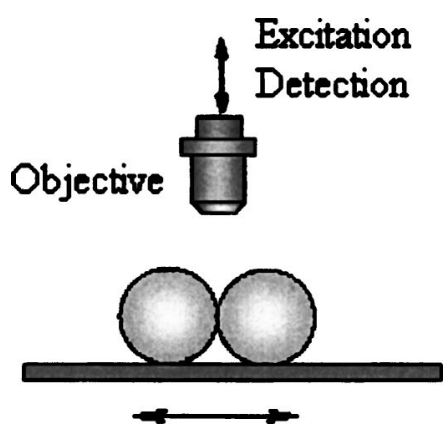

SCHEME 1. Experimental geometry for scanning PM along the longitudinal axis.

For a given excitation and detection configuration, we did not observe any new peaks in PL spectra of the PM (Fig. 2) as compared to that of single spheres (Fig. 1) except for weak broad features on the long-wavelength wings of the $\mathrm{TM}_{13}^{1}$ and $\mathrm{TM}_{14}^{1}$ modes [indicated by arrows in Fig. 2(b)]. These peaks are weak evidence of intermode coupling in PM. The pronounced double structure, with the intensity distribution dependent on the excitation position (Fig. 2), is just a result of superposition of the uncoupled WGM of individual microspheres. In this case, the scattered or emitted field from one microsphere is the additional incident field for another microsphere with intensity decreasing as the excitation spot moves farther away from the contact point. The lack of strong intermode coupling in this configuration is not surprising because the coupling between electromagnetic

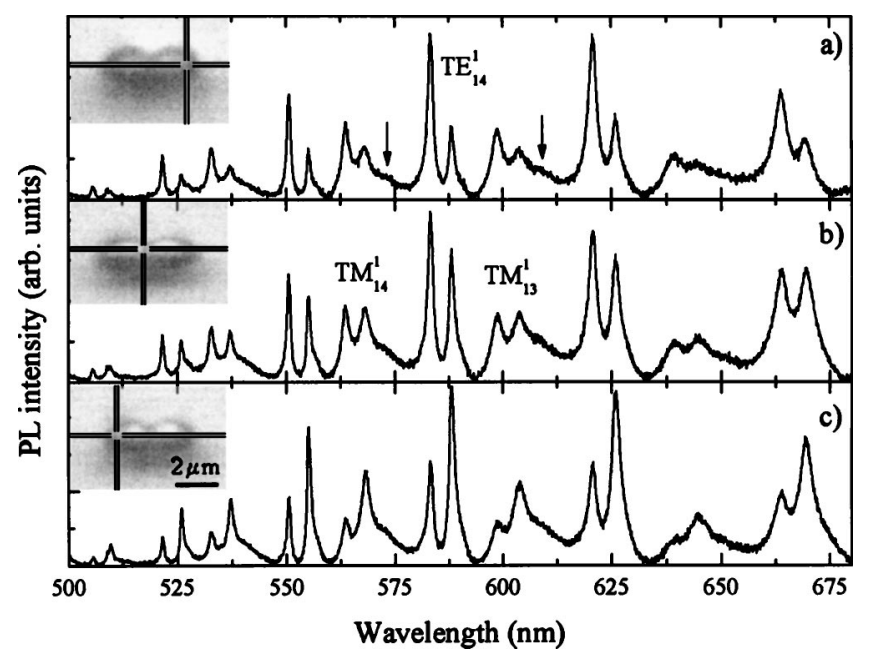

FIG. 2. Micro-PL spectra of a PM comprising two interacting spheres, with excitation and detection at three different positions along its longitudinal axis. Insets show microscope images of the PM with the crosshairs indicating the excitation-detection position. Background PL has been subtracted in all the spectra presented in order to show more clearly the WGM structure.

fields of the spheres is expected to be maximum in the direction parallel to the PM axis, when some of the WGM orbitals lie in the same plane. ${ }^{7}$ However, the observed redistribution of intensity between the components of WGM double structure clearly demonstrates the propagation of the light along the PM and possibility of wavelength switching in the PM, which can be used in optical interconnection devices. Clearly, the spectral position and the separation between components of the double PL structure (see, for example, spectral region of $\mathrm{TE}_{14}^{1}$ modes in Fig. 2) can be easily controlled by selecting microspheres of appropriate size.

Even with weak evidence of intermode coupling in the PM obtained from the steady-state PL spectrum, we can expect corresponding modification of the emission lifetime when compared with that of a single microsphere. To obtain further insight into the recombination dynamics in PM, we examined the time-resolved intensity decay of single and coupled spheres using the single-photon counting technique. In both cases decay curves were found to be complex (Fig. 3 ), indicating a number of widely spaced decay times. At least three decay times were needed to achieve a reasonable fit to the data, with decay times ranging from 1.3 to $15 \mathrm{~ns}$ and from 0.6 to $8.7 \mathrm{~ns}$ for single spheres and PM, respectively. It was predicted theoretically ${ }^{6}$ and demonstrated experimentally ${ }^{5,7,11}$ that the coupling of the photon modes in a PM can cause a narrow resonance of an individual microsphere to split into two modes of lower quality factor, implying a larger linewidth and smaller photon lifetime. Although at first glance our experimental data show the same behavior, the observed difference in lifetime is too big to be explained by a mode coupling effect alone. We have also observed more than a tenfold increase in the PL decay times of the PM with increasing detection wavelength from spectral position corresponding to the $\mathrm{TM}_{13}^{1}$ mode to spectral region of the $\mathrm{TM}_{12}^{1}$ mode (Fig. 3, curve 3). This increase of lifetime is much higher than that observed for a planar film of closepacked NCs (Ref. 18) or for a single microsphere. We be- 


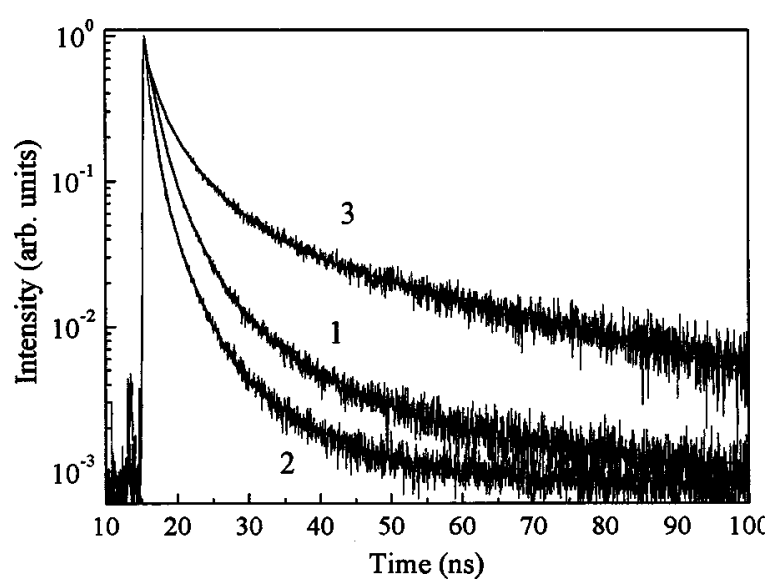

FIG. 3. Time-dependent PL intensity decays of a single CdTe/MF microsphere (1) and a PM with detection wavelength $\lambda=590 \mathrm{~nm}$ (2) and the PM with detection wavelength $\lambda=650 \mathrm{~nm}$ (3). Results of three-exponential analysis of decay curves are shown by thick lines.

lieve that optical feedback via the WGM of spherical microcavities can provide an increased probability of energy transfer to the emitting species. ${ }^{19}$ Therefore, in our case the observed dependence of lifetime on detection wavelength can be attributed to enhanced energy transfer between NCs of different sizes in a photonic molecule, providing faster decay time when compared with single microsphere PL response measured at the same detection wavelength.

As predicted in Ref. 7, controllable alignment geometry of the PM is crucial in order to observe the strong coupling between the spheres. The signal from the coupled microsphere modes is expected to be more pronounced when the incident light propagates parallel to the longitudinal axis of the PM. In order to control the alignment of the spheres constituting PM we utilized a polystyrene substrate containing a three-dimensionally ordered array of pores of $\sim 5 \mu \mathrm{m}$ in size prepared through a thermocapillary convection. ${ }^{20}$ The surface layer of the substrate contains an array of open microwells of 3- to $5-\mu \mathrm{m}$ depth. Only one pair of the $2-\mu \mathrm{m}$ microspheres can be accommodated within each microwell, and the axis of the PM is close to the surface normal. In the experiments detailed here, MF/CdTe microspheres were deposited on top of the porous substrate and then manipulated into position with a tapered optical fiber tip attached to a mechanical translation stage. Schematic excitation and detection geometry and microscope image of the PM in the well are presented in Fig. 4.
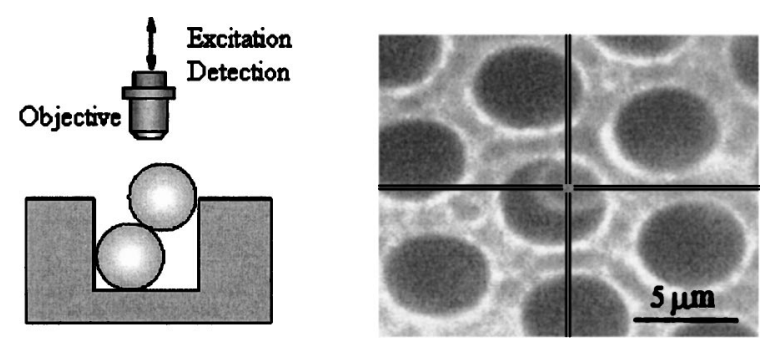

a)

b)

FIG. 4. Excitation and detection geometries (a) and microscope image of the PM in the microwell (b). The cross indicates the excitation position.

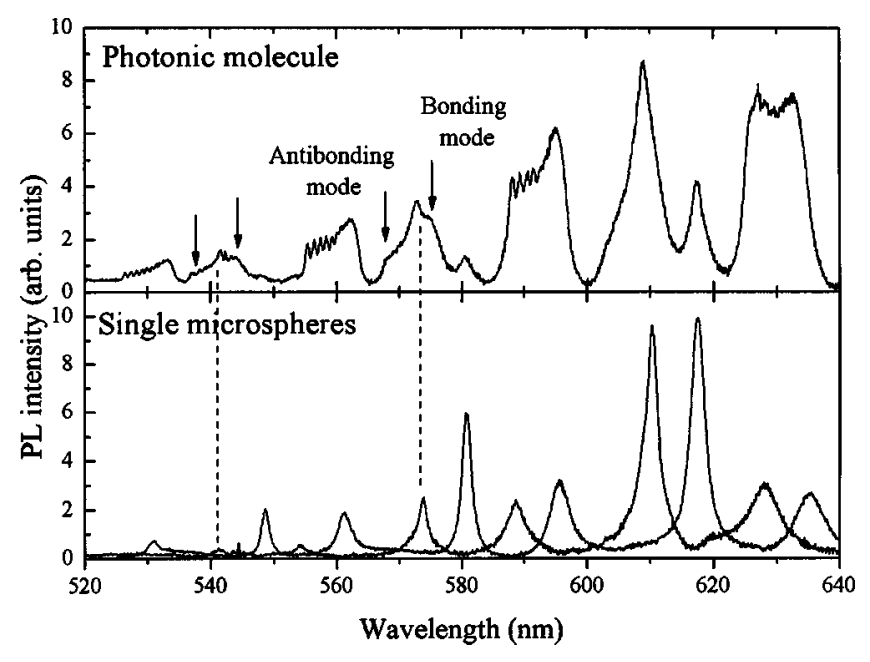

FIG. 5. Comparison of PL spectra of a PM accommodated in a microwell (top) and PL spectra of noninteracting microspheres (bottom). Arrows indicate the coupled modes.

The WGM structure in the PL spectra of single microspheres was found to be practically unaffected by the microporous substrate, except for a very small broadening of the WGM resonance lines. This can be understood by taking into account the difference in size between the microspheres and the microwell, and the interaction between the microwell walls and the microsphere is sufficiently small that the $Q$ value of the WGM is essentially preserved.

Figure 5 shows the PL spectrum of a PM accommodated in a microwell and the spectra of the individual microspheres prior to being manipulated into the microwell. The presented PL spectra clearly revealed two major features unique to strong coherent coupling between the photonic states of the two microspheres forming the PM. First, the appearance of the two satellites (indicated by arrows in Fig. 5) can be interpreted as a result of the formation of $\mathrm{BN}$ and $\mathrm{ABN}$ orbitals in the PM (Ref. 5) with the "ABN" peak observed at a lower wavelength than the "BN" one. The high PL efficiency of CdTe NCs and coupling of the electronic transitions of NCs to the resonances of PM allows us to detect the $\mathrm{BN}$ and $\mathrm{ABN}$ branches in a wide spectral region (520-600 nm). Secondly, one can clearly see a number of narrow peaks ( $m$ resonances) grouping in the spectrum for the PM on the short-wavelength side of the TE and TM resonances, which are due to the presence of $m \neq \pm 1$ components.

The number of experimentally observed peaks increases with the angular mode number both for TE and TM modes and is very close to the $n$ value, although never in excess of $n$ (Fig. 5). It was found that the spacing between the $m$ resonances and their intensity decreases significantly when approaching the long-wavelength limit. It should be noted that a similar effect in the case of a single microsphere can be caused by a nonspherical shape of the microparticle ${ }^{21}$ or by deformation of a microsphere. ${ }^{22-24}$ The first effect can be ruled out because of the extremely small size deviation in our samples. In the second case, lifting of degeneracy should be observed for all WGM without exception. This is in contrast to our case, where $m$ resonances have been only observed in the region of $\mathrm{ABN}$ modes of PM, where the effect 
of uncoupled WGM is minor. Furthermore, we have analyzed values of spacing between $m$ resonances depending on the eccentricity of single microspheres using the approach proposed in Ref. 21 . We found these values to be totally irreconcilable with our experimental data throughout the entire spectral region.

The deconvolution of the line shape of the $m$ resonances of the PM using Lorenzian functions reveals the most remarkable experimental fact: the $Q$ factor of $m$ resonances in the spectra of the PM $(\sim 2000)$ (and therefore lifetime, $\tau$, of a photon in the resonant modes) exceeds the $Q$ value $(\sim 800)$ and photon lifetime of individual spheres across the whole spectral region. This fact, along with the estimated value of BN/ABN splitting $(\sim 6-8 \mathrm{~nm})$, implies a possibility for the development of new PM-based photonic devices such as an optical delay line with controllable spectral and temporal tunability, which can be highly useful for a variety of applications in optical communication systems.

It is well known ${ }^{25,26}$ that microcavity-induced energytransfer rate depends on a number of parameters such as the length of the cavity, the positions of donor/acceptor, the polarization of dipoles, and the quality factor of the cavity modes which are in resonance with the electromagnetic field emitted by the excited dipoles. It is conceivable that the higher $Q$ factor of $m$ resonances observed in steady-state PL spectra of the PM causes an increase in the strength of dipole-dipole interaction and correspondingly an enhancement of the PL decay rate in comparison to that of the single microsphere.

\section{SUMMARY}

We have experimentally investigated the optical modes in a PM fabricated from two spherical microcavities coated with a thin shell of CdTe NCs. The observed lifetime increase is attributed to enhanced energy transfer between NCs of different sizes in a PM. PL spectra clearly reveal two major features unique to strong coherent coupling between the photonic states of the two microspheres forming the PM: the splitting of the WGM resonances originating from the $\mathrm{BN}$ and $\mathrm{ABN}$ branches of the photonic states and the fine structure of very sharp peaks resulting from lifting of the WGM degeneracy with respect to the azimuthal index. Our observation of coherently coupled modes in the PM accommodated in a microporous matrix shows the possibility of engineering new high- $Q$ devices with strong photon confinement.

\section{ACKNOWLEDGMENTS}

This work was supported by the Science Foundation Ireland under Grant Nos. 02/IN.1/I47 and 00/PI.1/C077A.2 and by the Deutsche Forschungsgemeinschaft through the SPP "Photonic Crystals."

${ }^{1}$ K. J. Vahala, Nature (London) 424, 839 (2003).

${ }^{2}$ Optical Processes in Microcavities, edited by R. K. Chang and A. J. Campillo (World Scientific, Singapore, 1996).

${ }^{3}$ E. Lidorikis, M. M. Sigalas, E. N. Economou, and C. M. Soukoulis, Phys. Rev. Lett. 81, 1405 (1998).

${ }^{4}$ S. Arnold, J. Comunale, W. B. Whitten, J. M. Ramsey, and K. A. Fuller, J. Opt. Soc. Am. B 9, 819 (1992).

${ }^{5}$ M. Bayer, T. Gutbrod, J. P. Reithmaier, A. Forchel, T. L. Reinecke, P. A. Knipp, A. A. Dremin, and V. D. Kulakovskii, Phys. Rev. Lett. 81, 2582 (1998).

${ }^{6}$ K. A. Fuller, Appl. Opt. 30, 4716 (1991)

${ }^{7}$ T. Mukaiyama, K. Takeda, H. Miyazaki, Y. Jimba, and M. KuwataGonokami, Phys. Rev. Lett. 82, 4623 (1999).

${ }^{8}$ H. Miyazaki and Y. Jimba, Phys. Rev. B 62, 7976 (2000).

${ }^{9}$ S. Arnold, A. Ghaemi, P. Hendrie, and K. A. Fuller, Opt. Lett. 19, 156 (1994).

${ }^{10}$ Y. Hara, T. Mukaiyama, K. Takeda, and M. Kuwata-Gonokami, Opt. Lett. 28, 2437 (2003).

${ }^{11}$ M. D. Barnes, S. M. Mahurin, A. Mehta, B. G. Sumpter, and D. W. Noid, Phys. Rev. Lett. 88, 015508 (2002).

${ }^{12}$ N. Gaponik, D. V. Talapin, A. L. Rogach, K. Hoppe, E. V. Shevchenko, A. Kornowski, A. Eychmüller, and H. Weller, J. Phys. Chem. B 106, 7177 (2002).

${ }^{13}$ Y. P. Rakovich, J. F. Donegan, N. Gaponik, and A. L. Rogach, Appl. Phys. Lett. 83, 2539 (2003).

${ }^{14}$ A. S. Susha et al., Colloids Surf., A 163, 39 (2000).

${ }^{15}$ Y. P. Rakovich, L. Yang, E. M. McCabe, J. F. Donegan, T. Perova, A. Moore, N. Gaponik, and A. Rogach, Semicond. Sci. Technol. 18, 914 (2003).

${ }^{16}$ A. G. Ryder, T. J. Glynn, M. Przyjalgowski, and B. Szczupak, J. Fluoresc. 12, 177 (2002).

${ }^{17}$ J. A. Stratton, Electromagnetic Theory (McGraw-Hill, New York, 1941).

${ }^{18}$ T. Franzl, D. S. Koktysh, T. A. Klar, A. L. Rogach, and J. Feldmann, Appl. Phys. Lett. 84, 2904 (2004).

${ }^{19}$ L. M. Folan, S. Arnold, and S. D. Druger, Chem. Phys. Lett. 118, 322 (1985).

${ }^{20}$ M. Srinivasarao, D. Collings, A. Philips, and S. Patel, Science 292, 79 (2001).

${ }^{21}$ H. M. Lai, P. T. Leung, K. Young, P. W. Barber, and S. C. Hill, Phys. Rev. A 41, 5187 (1990).

${ }^{22}$ V. V. Vassiliev, V. L. Velichansky, V. S. Ilchenko, M. L. Gorodetsky, L. Hollberg, and A. V. Yarovitsky, Opt. Commun. 158, 305 (1998).

${ }^{23}$ V. S. Ilchenko, P. S. Volikov, V. L. Velichansky, F. Treussart, V. LefevreSeguin, J. M. Raimond, and S. Haroche, Opt. Commun. 145, 86 (1998).

${ }^{24}$ W. von Klitzing, R. Long, V. S. Ilchenko, J. Hare, and V. Lefevre-Seguin, Opt. Lett. 26, 166 (2001).

${ }^{25}$ P. Andrew and W. L. Barnes, Science 290, 785 (2000).

${ }^{26}$ C. E. Finlayson, D. S. Ginger, and N. C. Greenham, Chem. Phys. Lett. 338, 83 (2001). 